\title{
Boundary time crystals
}

\author{
F. Iemini,${ }^{1}$ A. Russomanno, ${ }^{2,1}$ J. Keeling, ${ }^{3}$ M. Schirò, ${ }^{4}$ M. Dalmonte, ${ }^{1}$ and R. Fazio ${ }^{1,2}$ \\ ${ }^{1}$ ICTP, Strada Costiera 11, 34151 Trieste, Italy \\ ${ }^{2}$ NEST, Scuola Normale Superiore \& Istituto Nanoscienze-CNR, I-56126, Pisa, Italy \\ ${ }^{3}$ SUPA, School of Physics and Astronomy, University of St Andrews, St Andrews, KY16 9SS, United Kingdom \\ ${ }^{4}$ Institut de Physique Théorique, Universitée Paris Saclay, CNRS, CEA, F-91191 Gif-sur-Yvette, France
}

(Dated: June 12, 2018)

\begin{abstract}
In this work we introduce boundary time-crystals. Here continuous time-translation symmetry breaking occurs only in a macroscopic fraction of a many-body quantum system. After introducing their definition and properties, we analyse in detail a solvable model where an accurate scaling analysis can be performed. The existence of the boundary time crystals is intimately connected to the emergence of a time-periodic steady state in the thermodynamic limit of a many-body open quantum system. We also discuss connections to quantum synchronisation.
\end{abstract}

Introduction - Spontaneous symmetry breaking is a cornerstone of physics and occurs at all energy scales, in cosmology and high-energy physics as well as in condensed matter. Thermal or quantum fluctuations can drive a system into a state that breaks, in the thermodynamic limit, some of the symmetries present in its (thermo)-dynamical potentials $[1,2]$. Can timetranslation invariance be spontaneously broken? The possible existence of time crystals, first addressed by Wilczek in [3], prompted an intense discussion [4-7]. A no-go theorem [9] ruled out the existence of time-crystals in thermal equilibrium in cases for which the energy is the only constant of motion. The situation may be different in the presence of additional extended conserved quantities [10], such as in superfluids [11] where time-crystalline behavior was discussed in [12]. Ordering in time can also occur, however, under non-equilibrium conditions (e.g. by preparing the system in an excited state [13]).

An important step forward in our understanding of spontaneous time-translational invariance has been achieved in $[8,14,15]$ where Floquet time crystals, a.k.a. $\pi$-spin glasses, were introduced. The dynamics of these systems, subject to a periodic driving, is characterised by observables which oscillate at a multiple of the driving period. Hence they break the discrete time-translation symmetry imposed by the external drive. Floquet time crystals were intensively explored from a theoretical point of view in [16-22] and very recently experimentally observed $[23,24]$. A comprehensive review on time crystals can be found in [25].

Here we predict a novel form of time-translation symmetry breaking: continuous Boundary Time-Crystals (BTCs). In the BTC phase, symmetry breaking appears in a (macroscopic) fraction of the system. Moreover a BTC breaks the continuous time-translation symmetry, i.e. the system self-organises in a time-periodic pattern with a period which only depends on its coupling constants. The idea borrowed from surface critical phenomena [26] offer a very intuitive way to visualise BTCs. Only the surface, representing the portion of the system where time crystalline behaviour appear, is ordered. The rest
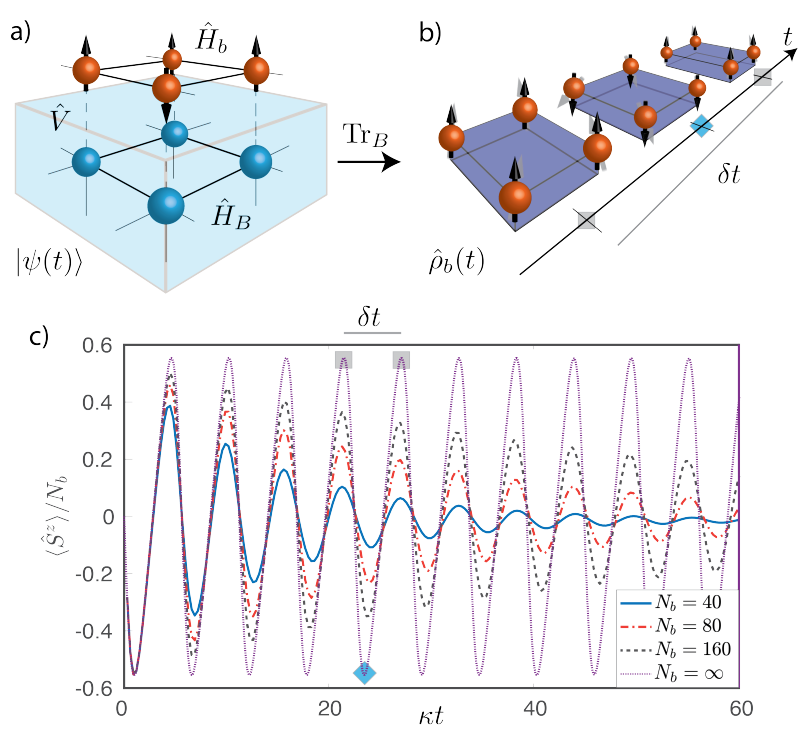

Figure 1. (Upper panel) A sketch of a boundary time crystal. The system is composed by a bulk (B) and a boundary (b) interacting trough an interaction term $\hat{V}$. The Hamiltonian of the system is time-independent. After tracing out the degrees of freedom of the bulk, the dynamics of the boundary is described by the reduced density matrix $\hat{\rho}_{\mathrm{b}}$. In the time-crystal phase, the behaviour of collective variables will show persistent oscillations in the thermodynamic limit. (Lower panel) The boundary magnetisation of the BTC model discussed in the paper is shown as a function of time, for different boundary sizes. In the asymptotic condition, spontaneous symmetry breaking appears in persistent oscillations when $N_{\mathrm{b}} \rightarrow \infty$.

of the system, the bulk remains time-translationally invariant, see Fig. 1. We will give a more precise meaning to this picture in the following of the paper where we will show that BTCs are intimately connected to the existence of periodic motion in the steady state of open quantum many-body systems.

Boundary time crystals - The emergence of a BTC can be understood using the sketch given in Fig. 1 (top panels). A $d$-dimensional quantum many-body system is governed by a time-independent Hamiltonian $\hat{H}=$ 
$\hat{H}_{\mathrm{B}}+\hat{H}_{\mathrm{b}}+\hat{V}$, with bulk and boundary systems $\hat{H}_{\mathrm{B}}$ and $\hat{H}_{\mathrm{b}}$, respectively, and an interaction term $\hat{V}$. Denoting as $N_{b}\left(N_{B}\right)$ the degrees of freedom for the boundary (bulk) systems, we consider the case in which a macroscopic fraction of the universe, the system $\left(N_{b} \rightarrow \infty\right)$, breaks spontaneously time-translational invariance. The thermodynamic limit is performed with $N_{b}, N_{B} \rightarrow \infty$, with the ratio $N_{b} / N_{B} \rightarrow 0$. In other words, it is a macroscopic system, but still small/infinitesimal compared to the global system. This scaling is the crucial feature in defining a boundary phenomenon. The precise identification of the boundary layer (e.g. the nature or any notion of spatial locality for its degrees of freedom) is thus irrelevant for our purposes. The whole system evolves according to the Schrödinger equation $|\psi(t)\rangle=e^{-i \hat{H} t}|\psi(0)\rangle$, with $|\psi(0)\rangle$ the initial state of the quantum system. The boundary is fully characterised by the reduced density matrix $\hat{\rho}_{\mathrm{b}}=\operatorname{Tr}_{\mathrm{B}}(|\psi(t)\rangle\langle\psi(t)|)$ obtained by tracing out the bulk degrees of freedom. Its dynamics is governed by a completely positive, trace-preserving, map $\hat{\mathcal{L}}$ with

$$
\frac{d}{d t} \hat{\rho}_{\mathrm{b}}=\hat{\mathcal{L}}\left[\hat{\rho}_{\mathrm{b}}\right]
$$

Time-translation symmetry breaking at the boundary appears as a non-trivial time-dependence of a (macroscopic) boundary order parameter $\hat{O}_{\mathrm{b}}$, occurring only in the thermodynamic limit. For infinitely large times its expectation oscillates, $\lim _{N_{\mathrm{b}}, N_{\mathrm{B}} \rightarrow \infty} \operatorname{Tr}\left[\hat{O}_{\mathrm{b}} \hat{\rho}_{\mathrm{b}}\right]=f(t)$ where $f(t)$ is a time-periodic function. The definition of BTC closely follows the one for the standard time crystals $[9,16,17]$. The only, crucial, difference is that here the order parameter is defined at the boundary.

The resulting physical picture is exemplified in Fig. 1, taking a magnetic system as an illustration. In this example a macroscopic magnetisation builds up at the surface of a sample. The magnetisation shows persistent oscillations even though the dynamics of the whole system is governed by a time-independent Hamiltonian. In Fig. 1 the boundary and the bulk are represented with different symbols in order to stress that they may be described by different degrees of freedom. Notice that the terms bulk and boundary are used here to easily visualise the mechanism of spontaneous symmetry breaking and suggest an intriguing connection with boundary critical phenomena. What is really implied in the construction above is that ordering in time occurs only in a macroscopic fraction of the many-body system under consideration, rather than in the whole bulk.

The boundary nature of time-translation symmetry breaking in BTC has a number of important implications. First of all, the reduced density matrix $\hat{\rho}_{\mathrm{b}}$ in the steady state will be generically non-thermal, hence the no-go theorem [9] does not apply: a Hamiltonian system can spontaneously break time-translation symmetry as a boundary phase. Furthermore, given the well known correspondence of the dissipative dynamics in Eq.(1) and a unitary dynamics governed by a time-independent Hamiltonian on an enlarged system (see e.g. [27]), the BTC appears tightly linked to the existence of a timeperiodic steady state in an open quantum many-body system, appearing though only for $N_{\mathrm{b}} \rightarrow \infty$. In order to discuss concrete examples we focus on boundary systems described by Markovian maps, and comment further below about more general dissipative maps.

The evolution of the boundary in the Markovian case is described by a master equation where the Liouvillian operator $\hat{\mathcal{L}}[\cdot]$ has Lindblad form $[27], \hat{\mathcal{L}}[\cdot]=$ $\sum_{\alpha}\left\{\hat{\ell}_{\alpha} \cdot \hat{\ell}_{\alpha}^{\dagger}-\frac{1}{2}\left\{\hat{\ell}_{\alpha}^{\dagger} \hat{\ell}_{\alpha}, \cdot\right\}\right\}$ with $\hat{\ell}_{\alpha}$ the Lindblad operators [33]. The emergence of a time-crystal behaviour in the long-time dynamics of the system is hidden in the properties of the Liouvillian operator in the thermodynamic limit. In the BTC phase one should expect: i) a vanishing gap in the real part of the Liouvillian eigenvalues, making the non-equilibrium steady state subspace degenerate in the thermodynamic limit with time-dependent coherences decaying over an infinite time-scale; ii) a non-zero imaginary part for some Liouvillian eigenvalues in such subspace in order to induce non-trivial oscillations. The main question now is to find a many-body system that displays the above mentioned properties. Below we will present a model of a BTC.

A BTC model - We will show that a boundary time crystal appears in a model used to describe cooperative emission in cavities (see [28-32]). The boundary Hamiltonian $\hat{H}_{\mathrm{b}}=\omega_{0} \sum_{j} \hat{\sigma}_{j}^{x}$ consists in a collection of $1 / 2$-spins whose dynamics is governed by collective spin operators $\hat{S}^{\alpha}=\frac{1}{2} \sum_{j} \hat{\sigma}_{j}^{\alpha}$. The operators $\hat{\sigma}_{j}^{\alpha}(\alpha=x, y, z)$ are the Pauli matrices acting on the j-th spins, and $\omega_{0}$ is the coherent splitting. The terms $\hat{H}_{\mathrm{B}}$ and $\hat{V}$ (see the sketch in Fig. 1) have to be constructed in such a way to give a reduced dynamics at the boundary of the form

$$
\frac{d}{d t} \hat{\rho}_{\mathrm{b}}=i \omega_{0}\left[\hat{\rho}_{\mathrm{b}}, \hat{S}^{x}\right]+\frac{\kappa}{S}\left(\hat{S}_{-} \hat{\rho}_{\mathrm{b}} \hat{S}_{+}-\frac{1}{2}\left\{\hat{S}_{+} \hat{S}_{-}, \hat{\rho}_{\mathrm{b}}\right\}\right) .
$$

In the previous equation, the collective raising/lowering spin operators are given by $\hat{S}_{ \pm}=\hat{S}^{x} \pm \hat{S}^{y}, \kappa$ is the effective decay rate, and $S=N_{\mathrm{b}} / 2$ is the total spin. In the following the expectations of the observables are indicated as $\langle\cdot\rangle=\operatorname{Tr}\left[\cdot \hat{\rho}_{\mathrm{b}}\right]$.

The specific form of $\hat{H}_{B}$ generating the dynamics in Eq. (2) will play no role. It is possible to derive it [33]. In the Supplementary Material we discuss in details how such a construction can be made [34]. Moreover similar Liouvillian dynamics have been extensively considered in the context of atomic systems coupled to cavity modes. Typically, the scenario in which a model such as Eq. (2) arises involves a system periodically driven at a finite frequency, with a time dependent Hamiltonian. Depending on the specific driving, such an explicit time dependence can be usually gauged away: one can define a Hamiltonian leading to Eq. (2) which is time- 
independent in some specific choice of frame. As long as such a Hamiltonian exists, and is physical, our interpretation of the time-translation symmetry breaking as a boundary phenomenon of a closed quantum system is reasonable (see [34] for a detailed discussion). Moreover - as we are going to show - the BTC shows a time-dependent pattern whose period solely depends on the coupling constants of the system and which is in general incommensurate with the driving period: the system breaks a continuous symmetry, rather than a discrete one. The BTC is in apparent contradiction with the expectation that the density matrix of a system in contact with a single thermal reservoir attains a time-independent steady state [35]. The solution to this apparent paradox lies in the diverging boundary size, $N_{b} \rightarrow \infty$, which leads to a divergent decay time-scale for oscillations (see [34]), as we better discuss below.

The steady state diagram of the model has two distinct phases [28]. For $\omega_{0} / \kappa<1$, the total magnetisation is finite $\left\langle\hat{S}^{z}\right\rangle$. In the opposite case, $\omega_{0} / \kappa>1$, all spins align along the $x$-direction. More details are reported in [34].
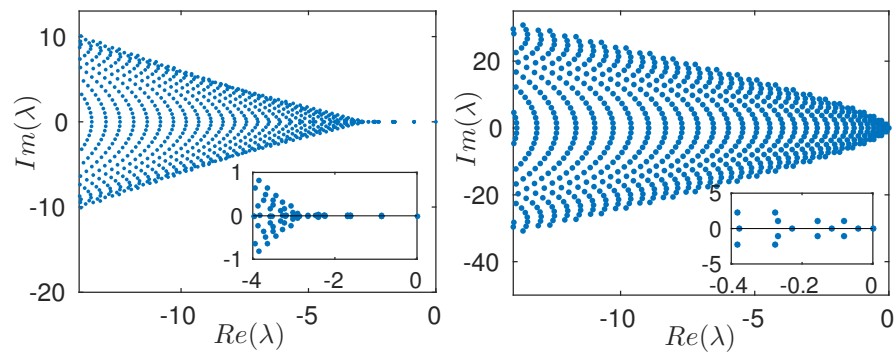

Figure 2. The eigenvalues $\lambda$ of the Liouvillian are shown in the weak dissipative case $\left(\omega_{0} / \kappa=1.5\right.$ - right panel) and in the strong dissipative one for $\left(\omega_{0} / \kappa=0.5\right.$ - left panel), in a system with $N_{\mathrm{b}}=36$ spins. The insets show a zoom over the eigenvalues with largest real part. The eigenvalues are plotted in units of $\kappa$.

The BTC appears for $\omega_{0} / \kappa>1$. Its emergence is embedded in the properties of the eigenvalues $\lambda$ of the Liouvillian $\hat{\mathcal{L}}$. The structure of the Liouvillian spectrum is indeed different in the two phases. While for $\omega_{0} / \kappa<1$ the spectrum is gapped (Fig. 2 left panel), and the eigenvalues with greatest values for their real part (i.e., the eigenvalues closest to zero, recalling that $\operatorname{Re}\left(\lambda_{j}\right) \leq 0$ ) have no imaginary values, for $\omega_{0} / \kappa>1$ the spectrum becomes gapless and the eigenvalues with greatest real part have a non zero imaginary part (see Fig. 2). The insets zoom on the spectrum emphasising the different behaviour in the two limits.

In order to obtain a quantitative picture of the development of the spontaneous symmetry breaking we perform a finite-size scaling analysis of the real and imaginary parts of the eigenvalues $\lambda$. In Fig. 3 (left panel) we analyse the real part of the Liouvillian spectrum. In the weak
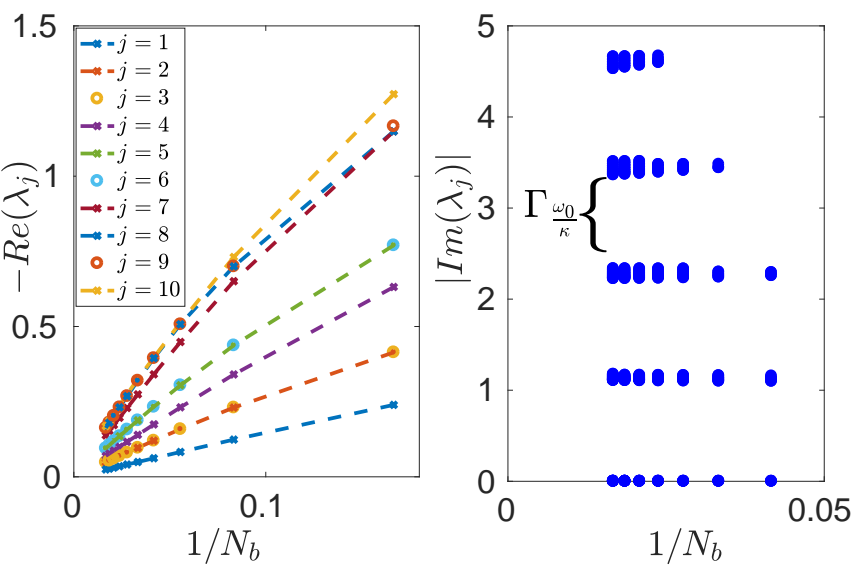

Figure 3. (Left) Finite size scaling for the real part of the Liouvillian eigenvalues in the BTC phase. The index $j$ labels the eigenvalues. The Liouvillian eigenvalues $\lambda_{j}$ are ordered as a function of their real part $\left(\left|\operatorname{Re}\left(\lambda_{j}\right)\right| \leq\left|\operatorname{Re}\left(\lambda_{j+1}\right)\right|\right.$, and $j=0$ has zero real part). In the $\omega_{0} / \kappa>1$ phase they scale to zero as a power-law of the inverse system size. (Right) The imaginary parts of the eigenvalues show a band structure, with a fundamental frequency separation $\Gamma_{\omega_{0} / \kappa}$. For fixed excitation thresholds (we only select $\lambda_{j}$ such that $\nu=j^{2} / N_{\mathrm{b}} \leq \epsilon$ ) the width of the bands remains finite in the thermodynamic limit (here we choose $\nu<0.025$ ). The widths of the bands tend to decrease as we constrain to lower excitation thresholds. The eigenvalues are plotted in units of $\kappa$.

dissipative case, the one of interest to us, the system is gapless, with the real part of the eigenvalues closing with the system size as a power law (at different rates). In Fig. 3 (right panel) we show the imaginary part of the Liouvillian spectrum. The imaginary eigenvalues of the low Liouvillian excitations are described by bands, separated by a fundamental frequency $\Gamma_{\omega_{0} / \kappa}$, which depends on the system parameters $\omega_{0} / \kappa$. These features of the real and imaginary parts are the key elements for the appearance of the BTC.

The magnetisation, for different numbers of lattice sites, is plotted in Fig. 1 (lower panel). The system is initialised in the pure state with all spins aligned along the $x$-direction. The oscillations decay for any finite size system, the associated time scale grows with the system size and diverges in the thermodynamic limit. This behaviour is independent of the initial conditions, as e.g., starting from thermal states or all spins aligned in different directions. Interestingly, the decay rate of the oscillations $\eta$ is related to the second excited eigenvalue of the Liouvillian, in our case, the lowest excited eigenvalue with non zero imaginary value (eigenvalues are ordered according to the absolute value of their real part). A quantitative analysis of the spontaneous symmetry breaking is obtained by looking at the Fourier transform of $\left\langle S^{z}(t)\right\rangle$ (see Fig. 4). By performing a spectral analysis, we see that the peaks appear at frequencies related to the separation between the bands shown in the right panel of 
Fig. 3. The peaks become sharper as the system size is increased. Most importantly, the decay rate $\eta$ goes to zero (right panel) as a power law $L^{-\beta}$ with the $\beta$ exponent dependent on the system parameters $w_{0} / \kappa$. The finite-size scaling shows that the persistent oscillations are associated to the spontaneous time-translation symmetry breaking because they occur only in the thermodynamic limit. In the example we have discussed, the thermodynamic limit incidentally coincides with an effective classical dynamics (the effective Planck's constant going to zero): this is true for instance in Fig. 4 and in Fig. 1 when $N_{\mathrm{b}} \rightarrow \infty$; details of the classical solution are discussed in [34].
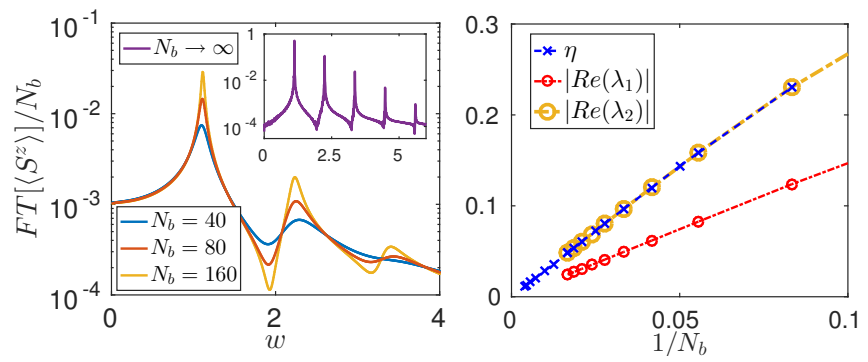

Figure 4. In the right panel we plot the decay rate of the oscillations of the magnetisation $\eta$ for distinct system sizes. In the same plot we compare $\eta$ with the eigenvalues with greatest real part - thus smallest absolute values for the real part of the Liouvillian. In the left panel we plot the Fourier transform of the average magnetisation (see lower panel in Fig. 1), highlighting the oscillation frequencies of the dynamics. The peaks are associated to the band separations in the imaginary part discussed in Fig. 3. The inset of the left panel is the solution in the thermodynamic limit where the oscillations persist indefinitely.

From the experimental point of view, a driven version of this model can be realised using an adapted Raman driving scheme [36] for cold atoms in an optical cavity, connecting two low lying states via an excited atomic state. Collective dissipation can be produced by using a bad cavity (large loss rate) combined with a single Raman drive: Purcell-enhanced Raman scattering leads to optical pumping of the atoms, described by the same collective dissipation considered here. Similarly, the Hamiltonian term $\hat{S}^{x}$ can be realised by a pair of drive lasers coupling the ground states via excited states.

The boundary time-crystal we discussed in the model of Eq.(2) is not an isolated point, but is robust to different perturbations. First of all, the time-crystalline phase appears in the whole region $\omega_{0} / \kappa>1$. Moreover it is stable if additional perturbations are added to the unitary part of the evolution. With a boundary Hamiltonian of the form $\hat{H}_{\mathrm{b}}=\omega_{0} \hat{S}^{x}+\omega_{x}\left(\hat{S}^{x}\right)^{2} / S+\omega_{z}\left(\hat{S}^{z}\right)^{2} / S$, the timecrystal is still present for a wide range of the parameters $\omega_{x}, \omega_{z} \neq 0$. In fact the $\omega_{x}$ term improves the stability of the time crystal, which is also present for small values of $\omega_{z}$; for $\omega_{z}$ above some threshold, time-translation symmetry breaking still exists but only for some initial conditions (see [34] for details). It is worth mentioning that robustness of a BTC phase refers to the persistence of a periodic evolution in the thermodynamic limit, and not necessarily to the rigidity of its period. The main difference with respect to Floquet systems is that there, since one is breaking a discrete symmetry, rigidity is intimately related to the period of the driving; instead, in our case, since the dynamics is $U(1)$ invariant, such timescale is not present, and the period of oscillation is allowed to change within the symmetry-broken phase. This is a direct analog of the fact that in spatial crystals, the spatial periodicity can be changed by changing the particle-particle interaction.

It is also relevant to consider perturbations of the dissipative part of the evolution, more specifically we focus on terms non-local in time (this is equivalent to considering a non-Markovian equation of motion). In order to have a physical bulk Hamiltonian, it must be bounded from below, and so it cannot have a truly flat density of states. This implies a finite memory timescale for the bath, but there is the possibility that this timescale can be neglected, being far smaller than all the other timescales in the system dynamics. This fact occurs if the lower bound on the bulk spectrum is at energies much lower than the frequencies of the system dynamics: in this case an approximate Markovian description holds and the use of a Markovian master equation is perfectly justified.

Other candidate systems for BTCs - An interesting model that should show the same phenomenology has been studied in [37]. Furthermore, manybody limit cycles have been already seen in model systems of optomechanical arrays [38], coupled cavity arrays $[39,40]$, interacting Rydberg atoms [41] and interacting spin-systems [42]. Also in these cases the underlying (bulk+boundary) Hamiltonian can be constructed, see Ref. [34]. In light of the analysis performed in the present work, these limit-cycles now might be classified as BTCs. It should be however kept in mind that a meanfield approximation, employed in these works, may be unable to support the very existence of limit cycles: it is not clear to which extend this phase would survive when fluctuations are included.

Other promising systems that it might be interesting to consider to seek for different forms of BTCs are dissipative topological systems. In this case the steady state may develop a degeneracy in the thermodynamic limit due to the presence of edge states [43, 44]. The existence of a BTC phase should emerge from the competition of the unitary and dissipative parts of the dynamics. Furthermore the robustness should be inherently linked to topological protection.

Finally a BTC, corresponding to a space-time ordering, represents in essence a synchronised dynamics in a many-body open quantum system. This hints to a very interesting and deep connection between time crystals 
and quantum synchronisation. Lately there has been an intense effort to characterise synchronisation in the quantum realm (see e.g., the review [45]). BTCs may offer a different perspective on this problem.

Conclusions - In this work we introduced boundary time crystals. In the same spirit as in the original definition given in [9], in the BTC phase the time-dependent order parameter appears only in a portion of the sample (at the boundary for simplicity). The phenomenon is analogous to surface critical phenomena. On looking at the reduced dynamics at the boundary, one observes that BTCs are intimately linked to the emergence of a periodic dynamics in some macroscopic observable of an open quantum many-body system. A crucial aspect of the whole picture is that the periodic motion should appear only in the thermodynamic limit. We proposed an example of a BTC phase in a solvable model where its existence can be confirmed without resorting to any approximation. We finally discussed that BTCs can also emerge from different mechanisms in topological systems.

While completing this manuscript, a few works appeared $[46,47]$ analysing discrete time crystal phenomena in periodically driven dissipative systems.

Acknowledgements - We acknowledge enlightening discussions with S. Denisov, J. Jin, L. Mazza, T. Prosen, and P. Zoller. This work was supported in part by "Progetti Interni - Scuola Normale Superiore" (A.R.), EU691 QUIC (R.F. and A.R.), CRF Singapore Ministry of Education (CPR-QSYNC 692) (R.F.), EPSRC program TOPNES (EP/I031014/1) (J.K.),

[1] N. Goldenfeld, Lectures on phase transitions and the renormalization group, (Addison Wesley, New York, 1992).

[2] S. Sachdev, Quantum Phase Transitions, (Cambridge University Press, Cambridge, 2000).

[3] F. Wilczek, Phys. Rev. Lett. 109, 160401 (2012).

[4] T. Li, Z.-X. Gong, Z.-Q. Yin, H. T. Quan, X. Yin, P. Zhang, L.-M. Duan, and X. Zhang, Phys. Rev. Lett. 109, 163001 (2012).

[5] P. Bruno, Phys. Rev. Lett. 110, 118901 (2013); ibid 111, 029301 (2013); ibid 111, 070402 (2013).

[6] P. Nozieres, EPL 103, 57008 (2013).

[7] G. E. Volovik, JETP Lett. 98, 491 (2013).

[8] K. Sacha, Phys. Rev. A 91, 033617 (2015).

[9] H. Watanabe and M. Oshikawa, Phys. Rev. Lett. 114, 251603 (2015).

[10] D. Huse (private communication).

[11] Hadrien Kurkjian, Yvan Castin and Alice Sinatra, Comptes Rendus Physique 17, 789 (2016).

[12] Nikolay V. Prokof'ev and Boris V. Svistunov, arXiv: 1710.00721.

[13] A. Syrwid, J. Zakrzewski, and K. Sacha, Phys. Rev. Lett. 119, 250602 (2017).

[14] D. V. Else, B. Bauer, and C. Nayak, Phys. Rev. Lett., 117, 090402 (2016).
[15] V. Khemani, A. Lazarides, R. Moessner, and S. L. Sondhi, Phys. Rev. Lett. 116, 250401 (2016).

[16] C. W. von Keyserlingk, V. Khemani, and S. L. Sondhi, Phys. Rev. B 94, 085112 (2016).

[17] V. Khemani, C. W. von Keyserlingk, S. L. Sondhi, arXiv:1612.08758.

[18] D. V. Else, B. Bauer, and C. Nayak, Phys. Rev. X 7, 011026 (2017).

[19] N.Y. Yao, A.C. Potter, I.-D. Potirniche, and A. Vishwanath, Phys. Rev. Lett. 118, 030401 (2017).

[20] W. W. Ho, S. Choi, M. D. Lukin, and D.A. Abanin, Phys. Rev. Lett. 119, 010602 (2017).

[21] B. Huang, Y.-H. Wu, W.V. Liu, arXiv:1703.04663.

[22] A. Russomanno, F. Iemini, M. Dalmonte, and R. Fazio, Phys. Rev. B 95, 214307 (2017).

[23] J. Zhang, P. W. Hess, A. Kyprianidis, P. Becker, A. Lee,J. Smith, G. Pagano, I. D. Potirniche, A. C. Potter, A.Vishwanath, N. Y. Yao, and C. Monroe, Nature 543, 217 (2017).

[24] S. Choi, J. Choi, R. Landig, G. Kucsko, H. Zhou, J.Isoya, F. Jelezko, S. Onoda, H. Sumiya, V. Khemani, C. von Keyserlingk, N. Y. Yao, E. Demler, and M. D. Lukin, Nature 543, 221 (2017).

[25] K. Sacha and Jakub Zakrzewski, arXiv:1704.03735.

[26] K. Binder, in Phase transitions and critical phenomena, Vol. 8 pag 2, C. Domb and J.L. Lebowitz Eds (Academic Press, New York, 1983).

[27] M. Nielsen and I. Chuang, Quantum Computation and Quantum Communication, (Cambridge University Press, Cambridge, 2000).

[28] J. Hannukainen and J. Larson, arXiv:1703.10238.

[29] D. F. Walls, P. D. Drummond, S. S. Hassan, and H. J. Carmichael, Prog. Theo. Phys. 64, 307 (1978).

[30] P. D. Drummond and H. J. Carmichael, Opt. Commun. 27, 160 (1978).

[31] R. R. Puri. and S. V. Lawande, Phys. Lett. A 72, 200 (1979).

[32] S. Schneider and G. J. Milburn, Phys. Rev. A 65, 042107 (2002).

[33] It is always possible to find a Hamiltonian whose dynamics is described by the given Lindblad equation. See e.g. A. M. Chebotarev, Math. Notes, 61, 510 (1997); M. Gregoratti, Commun. Math. Phys. 22, 181 (2001); J. Gough, Open Systems \& Information Dynamics, 22, 1550009 (2015); Javier Prior, Alex W. Chin, Susana F. Huelga and Martin B. Plenio, Phys. Rev. Lett. 105, 050404 (2010); Robert Rosenbach, Javier Cerrillo, Susana F. Huelga, Jianshu Cao and Martin B. Plenio, New. J. Phys. 18, 023035 (2016).

[34] Supplementary Material

[35] J. Cresser, J. Mod. Opt. 39, 2187 (1992)

[36] F. Dimer, B. Estienne, A. S. Parkins, and H. J. Carmichael, Phys. Rev. A 75, 013804 (2007)

[37] M. Hartmann, D. Poletti, M. Ivanchenko, S. Denisov, and P. Hänggi, arXiv:1606.03896.

[38] M. Ludwig and F. Marquardt, Phys. Rev. Lett. 111, 073603420 (2013).

[39] J. Jin, D. Rossini, R. Fazio, M. Leib, and M. J. Hartmann, Phys. Rev. Lett. 110, 163605 (2013);

[40] M. Schiró, C. Joshi, M. Bordyuh, R. Fazio, J. Keeling, and H. E. Türeci, Phys. Rev. Lett. 116, 143603 (2016).

[41] T. E. Lee, H. Häffner, and M. C. Cross, Phys. Rev. A 84, 416, 031402 (2011).

[42] C.-K. Chan, T. E. Lee, and S. Gopalakrishnan, Phys. 
Rev. A 91, 051601 (2015).

[43] S. Diehl, E. Rico, M. A. Baranov, and P. Zoller, Nat. Phys. 7, 971 (2011).

[44] F. Iemini, D. Rossini, R. Fazio, S. Diehl and L. Mazza, Phys. Rev. B 93, 115113 (2016).

[45] F. Galve, G. L. Giorgi, and R. Zambrini,
arXiv: 1610.05060

[46] Z. Gong, R. Hamazaki and M. Ueda, arXiv:1708.01472 (2017).

[47] R. R. W. Wang, B. Xing, G. G. Carlo and D. Poletti, arXiv:1708.09070 (2017). 